Relations industrielles

Industrial Relations

\title{
Organizations : Structure and Process, par Richard H. Hall, New Jersey, Englewood Cliffs, Prentice-Hall, Inc., 1972, 354 pp.
}

\section{Laurent Bélanger}

Volume 27, numéro 4, 1972

URI : https://id.erudit.org/iderudit/028353ar

DOI : https://doi.org/10.7202/028353ar

Aller au sommaire du numéro

Éditeur(s)

Département des relations industrielles de l'Université Laval

ISSN

0034-379X (imprimé)

1703-8138 (numérique)

Découvrir la revue

Citer ce compte rendu

Bélanger, L. (1972). Compte rendu de [Organizations : Structure and Process, par Richard H. Hall, New Jersey, Englewood Cliffs, Prentice-Hall, Inc., 1972, 354 pp.] Relations industrielles / Industrial Relations, 27(4), 799-800.

https://doi.org/10.7202/028353ar

Tous droits réservés (C) Département des relations industrielles de l'Université Laval, 1972
Ce document est protégé par la loi sur le droit d'auteur. L'utilisation des services d'Érudit (y compris la reproduction) est assujettie à sa politique d'utilisation que vous pouvez consulter en ligne.

https://apropos.erudit.org/fr/usagers/politique-dutilisation/ 
ouvrage à tendance sociale où l'auteur s'engage et révèle toute la philosophie qui l'anime.

La forme dans laquelle est rédigé le volume en respecte l'esprit; il utilise un style littéraire parfois même poétique, y glissant une pointe d'humour qui s'approche à certains moments du sarcasme.

La grande valeur de ce volume tient à l'esprit dans lequel il est présenté qui rejoint le nouveau courant de la direction d'entreprise. Son apport dans l'exercice du management réside en ce qu'il permettra aux administrateurs pris dans le quotidien de leurs tâches, de s'assurer un certain recul et de remettre en question des principes trop facilement acceptés ou tout au moins s'interroger sur l'optique dans laquelle ils l'ont été, ce qui en fait un livre de chevet tout indiqué pour les administrateurs soucieux de la bonne marche de leur organisation.

\section{Gaétan HUBERT}

Organizations : Structure and Process, by Richard H. Hall, New Jersey, Englewood Cliffs, Prentice-Hall, Inc., 1972, $354 \mathrm{pp}$.

C'est une contribution à la théorie des organisations dont l'objet spécifique consiste dans l'étude des structures et du fonctionnement des organisations et des relations que ces deux dimensions entretiennent avec un environnement donné. Comme on le sait, il existe plusieurs courants de pensée et de recherche dans ce champ aux frontières encore imprécises qu'est la psycho-sociologie des organisations. Cet ouvrage s'inscrit dans un cadre de référence tiré de l'approche sociotechnique en analyse organisationnelle avec un accent nettement sociologique. De fait, l'auteur opte avant tout pour une conception de l'organisation qui utilise la terminologie et la rationalité des systèmes ouverts, alors que les travaux de Weber et de Merton sur la bureaucratie envisagent l'organisation sous l'angle d'un système fermé, un agencement rationnel et calculé de moyens sans tenir compte des variables d'environnement.

L'auteur privilégie une perspective sociologique puisqu'il consacre la moitié de son ouvrage à l'étude des variables structurelles formelles et des relations qu'elles entretiennent en elles.

Ce n'est pas tellement la perspective retenue qui distingue ce volume des au- tres dans le même domaine. C'est plutôt l'effort qu'a fait l'auteur pour rapatrier sous des têtes de chapitres significatives une masse d'information tirée des recherches empiriques éparses effectuées au cours de la dernière décade sur l'identification et la mesure des variables structurelles qui caractérisent l'aspect formel de toute organisation sociale. Une attention spéciale est aussi accordée aux variables de fonctionnement ou processus tels que le pouvoir, le leadership, la prise de décision, les communications.

En introduction, l'auteur tente de définir sans y réussir complètement, les notions d'organisation sociale, de système social ouvert, de système social fermé. Ensuite, il présente le cadre de référence qu'il utilise au cours de la revue des travaux sur les organisations. Le deuxième chapitre fait état des différents essais de typologie ou classification des organisations sans arriver à en établir une qui soit satisfaisante. Le troisième chapitre consiste en une discussion sur la notion d'objectifs organisationnels en particulier, celle de l'efficacité. Encore là on saisit toute la difficulté de définir des objectifs qui soient opérationnels. $\mathrm{La}$ deuxième partie traite du degré de bureaucratisation des organisations en retenant trois grandes variables: la taille, la complexité et la formalisation. Les processus internes de fonctionnement tels que le pouvoir et le conflit, le leadership, la prise de décision et les communications font l'objet de la troisième partie. C'est cette section qui nous apparaît la plus faible. D'abord, parce qu'elle résume trop brièvement pour être compréhensible les travaux de recherche effectués sur le leadership et la prise de décision; ensuite, parce qu'elle ne réussit pas à présenter correctement le jeu des rapports interpersonnels qui sont au cœur de l'animation des structures. Il est probable, dans ce cas, qu'un compte-rendu commenté et trop poussé aurait forcé l'auteur à sortir un peu de son cadre d'analyse et à allonger indûment cette troisième partie. La structure et le fonctionnement d'une organisation entretiennent des rapports très étroits et réciproques avec les variables contextuelles telles que les conditions technologiques, légales, politiques, économiques, démographiques et culturelles : ceci constitue l'objet de la dernière partie. Beaucoup de recherches et d'efforts de conceptualisation se font actuellement sous cet aspect en 
nous apportant un éclairage nouveau et une compréhension plus élargie de la structure et du fonctionnement des organisations.

Dans l'ensemble, c'est un ouvrage bien structuré, de lecture facile, du moins pour celui qui possède quelques notions de base en sociologie et en administration des entreprises.

\section{Laurent BELANGER}

Race and Industrial Conflict, by Malcolm Rimmer, London, Heinemann Educational Books, 1972, $74 \mathrm{pp}$.

Cet ouvrage, concis et bien présenté, analyse les phénomènes d'intégration et de solidarité ethnique par le biais du conflit industriel. Il ne s'agit pas cependant d'une analyse exhaustive, ce qui est compréhensible puisque l'auteur présente une monographie.

Malcolm Rimmer étudie ici les effets d'unités de travail ethniques sur les relations de travail dans l'entreprise et plus précisément l'impact qu'elles ont sur les coutumes et les règles informelles.

Pour cette monographie portant sur un groupe de fonderies du Midlands, l'auteur a utilisé une démarche assez simple. D'une part, les deux thèmes principaux de l'étude sont: l'analyse du développement d'une rupture normative dans les relations de travail et la nature des relations conflictuelles dans l'industrie entre les immigrants de couleur, les travailleurs blancs et la direction. D'autre part, l'analyse des données empiriques est décomposée en quatre étapes: l'embauche d'un groupe d'immigrants de couleur dans des fonderies; la naissance d'un syndicalisme fort qui repose sur des liens de solidarité ethnique; le conflit entre les immigrants syndiqués, la direction et les travailleurs blancs; le développement de procédures formelles en vue d'éviter les conflits.

L'auteur montre qu'un des effets majeurs sur les relations de travail de l'emploi de travailleurs immigrés, constituant des groupes solidaires, est de modifier sensiblement les règles établies par la coutume et qui régissent néanmoins certains rapports de travail dans l'industrie britannique. La conséquence immédiate de cette rupture avec la coutume est une augmentation du conflit industriel et la recherche soit d'une plus grande formalisation des règles, soit de l'élaboration de nouvelles normes par les groupes ethniques et adaptées à leurs valeurs et à leurs coutumes. En effet, les règles industrielles qui découlent de la pratique et de la coutume sont plus instables que celles qui sont définies unilatéralement par la direction ou explicitées dans une convention collective. Les unes peuvent être communiquées et expliquées facilement car elles relèvent de décisions rationnelles, alors que les autres s'apprennent au contact de ceux qui les observent. Si les réseaux de communication ne fonctionnent pas les coutumes sont difficilement transmises, les groupes ethniques développent leur propre modèle de règles et il en résulte alors de véritables conflits sociaux. En fait, l'auteur montre que deux situations sont possibles: si les immigrants sont maintenus isolés, l'unité de travail constitue un groupe primaire stable où la culture de l'immigrant est préservée; si des réseaux de communication sont établis, les immigrants n'ont pas tendance à se regrouper dans des unités isolées, adoptent le comportement de leurs camarades de travail et, alors, ils sont partiellement intégrés.

Gilles LAFLAMME

\section{Les femmes dans la population active :} 1970, faits et données, Ottawa, Bureau de la main-d'œuvre féminine, Ministère du travail du Canada, 1971, $75 \mathrm{pp}$.

La participation des femmes à la population active attire de plus en plus d'attention. Cette publication du Ministère fédéral du travail en est un témoignage éloquent. Les femmes dans la population active est essentiellement descriptif de la situation de la main-d'œuvre féminine au Canada et dans ses diverses régions et des changements majeurs qu'elle a connus depuis 1960 . Cet ouvrage présente d'abord dix caractéristiques de la main-d'œuvre féminine canadienne. Cette synthèse est sûrement très utile et soulève à elle seule plusieurs sujets de recherche. Le reste de cette publication présente trente trois tableaux regroupés à l'intérieur de quatre catégories: les enquêtes mensuelles, employées et personnes à leur compte, gains et emploi en général et dans les industries relevant de l'autorité fédérale.

C'est sûrement un recueil de statistiques utile et bien fait. Cependant cette publication ne comble pas le besoin d'analyse en ce domaine.. Ce n'est qu'un outil de travail.

Jean SEXTON 